\title{
Ontological Foundations for Conceptual Part-Whole Relations: The Case of Collectives and Their Parts
}

\author{
Giancarlo Guizzardi \\ Ontology and Conceptual Modeling Research Group (NEMO), \\ Federal University of Espírito Santo (UFES), Vitória (ES), Brazil \\ gguizzardi@inf.ufes.br
}

\begin{abstract}
In a series of publications, we have employed ontological theories and principles to evaluate and improve the quality of conceptual modeling grammars and models. In this article, we advance this research program by conducting an ontological analysis to investigate the proper representation of types whose instances are collectives, as well as the representation of part-whole relations involving them. As a result, we provide an ontological interpretation for these notions, as well as modeling guidelines for their sound representation in conceptual modeling. Moreover, we present a precise qualification for the parthood relations of member-collective and subcollectivecollective in terms of formal mereological theories of parthood, as well as in terms of the modal meta-properties of essential and inseparable parts.
\end{abstract}

Keywords: ontological foundations for conceptual modeling, part-whole relations, representation of collectives.

\section{Introduction}

In recent years, there has been a growing interest in the application of Foundational Ontologies, i.e., formal ontological theories in the philosophical sense, for providing real-world semantics for conceptual modeling languages, and theoretically sound foundations and methodological guidelines for evaluating and improving the individual models produced using these languages.

In a series of publications, we have successfully employed ontological theories and principles to analyze a number of fundamental conceptual modeling constructs such as Types, Roles and Taxonomic Structures, Relations, Attributes, among others (e.g., $[1,2])$. In this article we continue this work by investigating a specific aspect of the representation of part-whole relations. In particular, we focus on the ontological analysis of collectives and the part-whole relations involving them. The focus on collectives is timely given the increasing recognition of the importance of finding well-founded manners to represent collectives in domains such as bioinformatics in which collectives and their parts abound $[3,4]$.

Parthood is a relation of fundamental importance in conceptual modeling, being present as a modeling primitive in practically all major conceptual modeling languages. Motivated by this, a number of attempts have been made to employ theories of different sorts to provide a foundation for part-whole relations. These initiatives 
fall roughly in three different classes: (i) proposals that employ classical ontological theories of parthood (Mereologies). In this class, there is a number of works in the literature that employ the ontological theory put forth by the philosopher Mario Bunge [5] typically accessed through its most popular adaptation termed the BWW ontology [6,7]; (ii) proposals that are based on research from linguistics and cognitive science in which different sorts of Meronymic relations are elaborated. Most contributions in this class are based on the theory developed by Winston, Chaffin and Herrmann (henceforth WCH) [8]. An example of a pioneering article in this class is [9]; (iii) proposals which define a number of so-called secondary properties which have been used to further qualify parthood relations [6]. These include distinctions which reflect different modal aspects of parthood reflecting different relations of dependence (e.g., generic versus existential dependence). An example is the distinction between essential and inseparable parthood in [2].

Despite their important contributions, there are significant shortcomings in the current scenario considering the aforementioned approaches. On the one hand, accounts of parthood solely based on WCH suffer from many difficulties inherited from the original theory. As discussed in [10,11], WCH's original taxonomy turned out to be overly linguistically motivated, focusing on the linguistic term part-of (and its cognates). In fact, as demonstrated by these authors, the six linguistically-motivated types of part-whole relation originally proposed in $\mathrm{WCH}$ give rise to only four distinct ontological types, namely: (a) subquantity-quantity: modeling parts of an amount of matter (e.g., alcohol-wine, gin-Martini, Chocolate-Toddy); (b) member-collective: modeling a collective entity in which all parts play an equal role w.r.t. the whole (e.g., tree-forest, card-deck, lion-pack); (c) subcollective-collective: modeling a relation between a collective and the subcollectives that provide further structure to the former (e.g., the north part of the black forest-black forest, the underage children of John-the children on John); (d) component - functional complex: modeling an entity in which all parts play a different role w.r.t. the whole, thus, contributing to the functionality of the latter (e.g., heart-circulatory system, engine - car).

On the other hand, conceptual modeling accounts of parthood based on BWW inherit the limitations of Bunge's original treatment of parthood in its most basic core. Mereology is a mature discipline with well-defined and formally characterized theories. These in fact form a lattice of theories such that there is not one single formal meaning of part in mereology but several alternative axiomatizations of parthood that extend each other. Mapping modeling primitives representing part-whole relations to these theories can indeed provide an important contribution to conceptual modeling. Firstly, in a direct manner because these theories can provide sound and fully characterized formal semantics for these relations. But, also because nowadays several authors have proposed codifications of different mereological theories by mapping them to different Description Logics, hence, providing a mechanism for automated reasoning with partonomies in conceptual models $[12,13]$. The negative point here is that Bunge's theory of parthood corresponds to the weakest theory in mereology. In fact a theory which is even considered to be too weak to count as a characterization for a true part-whole relation [14].

Finally, most current approaches limit themselves to analyze the relation between the whole and its parts. However, as discussed in [15], a conceptual theory of parthood should also countenance a theory of wholes, in which the relations that tie 
the parts of a whole together are also considered. To put it simply, the composite objects in which we are interested in conceptual modeling are not mere aggregations of arbitrary entities but complex entities suitably unified by proper binding relations.

This paper should then be seen as a companion to the publications in $[2,16]$ and [17]. In this research program, we have managed to show that the three classification schemes aforementioned, namely, the linguistic-cognitive meronymic distinctions, the mereological theories of parthood, and the so-called secondary properties are not orthogonal. In fact, each particular meronymic distinction in the first scheme commits to basic mereological properties, secondary properties, and even requires binding relations of specific kinds to take place between their parts. In [17], we have managed to show the interconnection between these classification schemes for the case of the subquantity-quantity relation. In a complementary form, we did the same in $[2,16]$ for the case of the component - functional complex relation. The objective of this paper is to follow the same program for the case of part-whole relations involving collectives, namely, the member-collective and the subcollective-collective relations. This paper is, thus, a substantial extension to the preliminary work reported in [18] in which only the member-collective relation is analyzed and in some of its aspects.

The remainder of this article is organized as follows. Section 2 reviews the theories put forth by classical mereology as well as its connection with modal secondary properties of parts and wholes. The section also discusses how these mereological theories can be supplemented by a theory of (integral) wholes. In section 3, we discuss collectives as integral wholes and present some modeling consequences of the view defended there. Moreover, we elaborate on some ontological properties of collectives that differentiate them not only from their sibling categories (quantities and functional complexes), but also from sets (in a set-theoretical sense). The latter aspect is of relevance since collectives as well as the member-collective and subcollective-collective relations are frequently taken to be identical to sets, set membership and the subset relation, respectively. In section 4 , we promote an ontological analysis of two partwhole relations involving collectives, clarifying on how these relations stand w.r.t. to basic mereological properties (e.g., transitivity, weak supplementation, extensionality) as well as regarding the modal secondary properties of essential and inseparable parthood. As an additional result connected to this analysis, we outline a number of metamodeling constraints that have been used to implement a UML modeling profile for representing collectives and their subparts in conceptual modeling. Section 5 presents final considerations of this paper.

\section{A Review of Formal Part-Whole Theories}

In practically all philosophical theories of parts, the relation of (proper) parthood (symbolized as <) stands for a strict partial ordering, i.e., an asymmetric (2) and transitive relation (3), from which irreflexivity follows (1):

$$
\begin{gathered}
\forall \mathbf{x} \neg(\mathbf{x}<\mathbf{x}) \\
\forall \mathbf{x}, \mathbf{y}((\mathbf{x}<\mathbf{y}) \rightarrow \neg(\mathbf{y}<\mathbf{x})) \\
\forall \mathbf{x}, \mathbf{y}, \mathbf{z}((\mathbf{x}<\mathbf{y}) \wedge(\mathbf{y}<\mathbf{z}) \rightarrow(\mathbf{x}<\mathbf{z}))
\end{gathered}
$$


These axioms amount to what is referred in the literature by the name of Ground Mereology $(M)$, which is the core of any theory of parts, i.e., the axioms (1-3) define the minimal (partial ordering) constraints that every relation must fulfill to be considered a parthood relation. As previously mentioned, Mario Bunge's mereological theory (Assembly Theory) corresponds to the axiomatization of Ground Mereology (with the only difference of assuming the existence of a null individual which is supposed to be part of everything else) [5]. Although necessary, these constraints are not sufficient, i.e., it is not the case that any partial ordering relation qualifies as a parthood relation. Most authors require an extra axiom termed the weak supplementation principle (WSP) (4) as constitutive of the meaning of part and, hence, consider (1-3) plus (4) (the so-called Minimal Mereology (MM)) as the minimal constraints that a mereological theory should incorporate $[14,19]$ :

$$
\forall \mathbf{x}, \mathbf{y}((\mathbf{y}<\mathbf{x}) \rightarrow \exists \mathbf{z}(\mathbf{z}<\mathbf{x}) \wedge \neg \operatorname{overlap}(\mathbf{z}, \mathbf{y}))
$$

Figure 1.a below illustrates this notion of weak supplementation. It shows that if $y$ is a part of $\mathrm{x}$ then there must exist another part of $\mathrm{x}$ which is disjoint from $\mathrm{y}$ (the "missing" part of $\mathrm{x}$ ). Without this "missing" part, what differentiates $\mathrm{y}$ and $\mathrm{x}$ ? Notice that $\mathrm{x}$ and $y$ are supposed to be different given that parthood is irreflexive (1). From a practical point of view, without WSP, models such as the one in figure 1.b cannot be deemed incorrect. Now, following that model, suppose an event $\mathrm{E}$ which is composed of one single subevent. Isn't this alleged part identical to the event E itself? In a sound model, events are either atomic or are composed of at least two disjoint subevents.
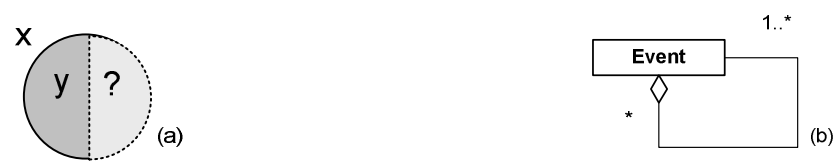

Fig. 1. Invalid situation (a) and invalid conceptual model (b) according to Minimum Mereology

There is an extension to MM that has then been created by strengthening the supplementation principle represented by (4). In this system, (4) is thus replaced by something termed the stronger supplementation principle (SSP). The resulting theory is named Extensional Mereology (EM). A known consequence of the introduction of SSP is that in EM we have that two objects are identical iff they have the same parts, i.e., SSP entails a mereological counterpart of the extensionality principle (of identity) in set theory. As a consequence, if an entity is identical to the (mereological) sum of its parts, thus, changing any of its parts changes the identity of that entity. Ergo, an entity cannot exist without each of its parts, which is the same as saying that all its parts are essential parts.

Essential parthood can be defined as a case of existential dependence between individuals, i.e., $\mathrm{x}$ is an essential part of $\mathrm{y}$ iff $\mathrm{y}$ cannot possibly exist without having that specific individual $\mathrm{x}$ as part [2]. This specific mode of existential dependence can also be defined from the part $\mathrm{x}$ to the whole $\mathrm{y}$. We say that $\mathrm{x}$ is an inseparable part of $\mathrm{y}$ iff $\mathrm{x}$ cannot possibly exist without being a part of that specific individual $\mathrm{y}$ [2]. A 
stereotypical example of an essential part of a car is its chassis, since that specific car cannot exist without that specific chassis (changing the chassis legally changes the identity of the car); A stereotypical example of an inseparable part of a living cell is its membrane, since the membrane cannot exist without being part of that particular cell. As discussed in depth in [2], essential and inseparable parthood play a fundamental role in conceptual modeling. However, it is not the case for all types of entities that all their parts are essential. In other words, although EM describes the basic meaning of parthood for some types of entities (e.g., quantities [17] and events [14]), this is not the case for entities of all ontological categories. In particular, as we have shown in [16], for functional complexes while some of their parts are essential (inseparable), not all of them are essential (inseparable). As discussed in section 4, EM is too strong a theory in this sense also for the case of the member-collective and the subcollectivecollective relations.

Classical mereological theories focus solely on the relation from the parts to the wholes. Thus, just like in set theories we can create sets by enumerating any number of arbitrary entities, in classical mereologies one can create a new object by summing up individuals that can even belong to different ontological categories. For example, in these systems, the individual created by the aggregation (termed mereological sum) of Noam Chomsky's left foot, the first act of Puccini's Turandot and the number 3, is an entity considered as legitimate as any other. However, as argued by [10], humans only accept the aggregation of entities if the resulting mereological sum plays some role in their conceptual schemes. To use an example: the sum of a frame, a piece of electrical equipment and a bulb constitutes a whole that is considered meaningful to our conceptual classification system. For this reason, this sum deserves a specific concept in cognition and a name in human language. The same does not hold for the sum of bulb and the lamp's base.

According to Simons [14], the difference between purely formal mereological sums and, what he terms, integral wholes is an ontological one, which can be understood by comparing their existence conditions. For sums, these conditions are minimal: the sum exists just when the constituent parts exist. By contrast, for an integral whole (composed of the same parts of the corresponding sum) to exist, a further unifying condition among the constituent parts must be fulfilled. A unifying condition or relation can be used to define a closure system in the following manner. A set B is a closure system under the relation $\mathrm{R}$, or simply, R-closure system iff

$$
\operatorname{cs}\langle\mathbf{R}\rangle \mathbf{B}=_{\text {def }}(\operatorname{cl}\langle\mathbf{R}\rangle \mathbf{B}) \wedge(\operatorname{con}\langle\mathbf{R}\rangle \mathbf{B})
$$

where (cl $\langle\mathbf{R}\rangle \mathbf{B})$ means that the set B is closed under R (R-Closed) and (con $\langle\mathbf{R}\rangle \mathbf{B})$ means that the set $\mathrm{B}$ is connected under $\mathrm{R}$ (R-Connected). R-Closed and R-Connected are then defined as:

$$
\begin{gathered}
\mathbf{c l}\langle\mathbf{R}\rangle \mathbf{B}==_{\text {def }} \forall \mathbf{x}((\mathbf{x} \in \mathbf{B}) \rightarrow((\forall \mathbf{y} \mathbf{R}(\mathbf{x}, \mathbf{y}) \vee \mathbf{R}(\mathbf{y}, \mathbf{x}) \rightarrow(\mathbf{y} \in \mathbf{B}))) \\
\operatorname{con}\langle\mathbf{R}\rangle \mathbf{B}==_{\text {def }} \forall \mathbf{x}((\mathbf{x} \in \mathbf{B}) \rightarrow(\forall \mathbf{y}(\mathbf{y} \in \mathbf{B}) \rightarrow(\mathbf{R}(\mathbf{x}, \mathbf{y}) \vee \mathbf{R}(\mathbf{y}, \mathbf{x})))
\end{gathered}
$$

An integral whole is then defined as an object whose parts form a closure system induced by what Simons terms a unifying (or characterizing) relation $\mathrm{R}$. 


\section{What are Collectives?}

According to $\mathrm{WCH}$, the main distinction between collectives and quantities is that the latter but not the former are said to be homeomeros wholes [8]. In simple terms, homeorosity means that the entity at hand is composed solely of parts of the same type (homo=same, mereos = part). The fact that quantities are homeomeros (e.g., all subportions of wine are still wine) causes a problem for their representation (and the representation of relationships involving them) in conceptual modeling. In order to illustrate this idea, we use the example depicted in figure 2.a below. In this model, the idea is to represent that a certain portion of wine is composed of all subportions of wine belonging to a certain vintage, and that a wine tank can store several portions of wine (perhaps an assemblage of different vintages). However, since Wine is homeomeros and infinitely divisable in subportions of the same type, if we have that a Wine portion $\mathrm{x}$ has as part a subportion $\mathrm{y}$ then it also has as part all the subparts of $\mathrm{y}$ [17]. Likewise, a wine tank storing two different "portions of wine" actually stores all the subparts of these two portions, i.e., it actually stores infinite portions of wine. In other words, maximum cardinality relations involving quantities cannot be specified in a finite manner. As discussed, for instance in [20], finite satisfiability is a fundamental requirement for conceptual models which are intended to be used in information systems. This feature of quantities, thus, requires a special treatment so that they can be property modeled in structural conceptual models. A treatment that does not take quantities to be mere aggregations (mereological sums) of subportions of the same kind but integral wholes unified by a characterizing relation of topological maximal self-connectedness [17].
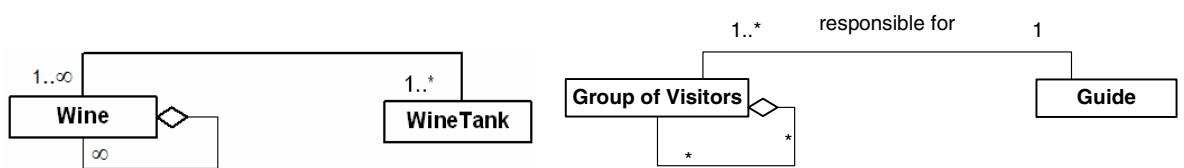

Fig. 2. Representations of a Quantity (a-left) and a Collective (b-right) with their respective parts in UML conceptual Models

As correctly defined by WCH, collectives are not homeomeros. They are composed of subparts parts that are not of the same kind (e.g., a tree is not forest). Moreover, they are also not infinitely divisible. As a consequence, a representation of a collection as a simple aggregation of entities (analogous to an enumerated set of entities) does not lead to the same complications as for the case of quantities. Take, for instance, the example depicted in figure 2.b, which represents a situation analogous to the one of figure 2.a. Different from the former case, there is no longer the danger of an infinite regress or the impossibility of specifying finite cardinality constraints. In figure 2.b, the usual maximum cardinality of "many" can be used to express that a group of visitors has as parts possibly many other groups of visitors and that a guide is responsible for possibly many groups of visitors.

Nonetheless, in many examples (such as this one), this model of figure 2.b implies a somewhat counterintuitive reading. In general, the intended idea is to express that, 
for instance, John as a guide, is responsible for the group formed by \{Paul, Marc, Lisa $\}$ and for the other group formed by $\{$ Richard, Tom $\}$. The intention is not to express that John is responsible for the groups $\{$ Paul, Marc, Lisa $\},\{$ Paul, Marc $\},\{$ Marc, Lisa\}, $\{$ Paul, Lisa $\}$, and $\{$ Richard, Tom\}, i.e., that being responsible for the group $\{$ Paul, Marc, Lisa\}, John should be responsible for all its subgroups. A simple solution to this problem is to consider groups of visitors as maximal sums, i.e., groups that are not parts of any other groups. In this case, depicted in figure 3, the cardinality constraints acquire a different meaning and it is no longer possible to say that a group of visitors is composed of other groups of visitors in this technical sense.

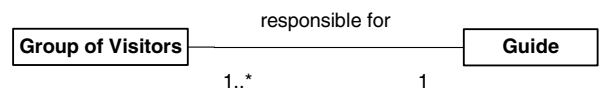

Fig. 3. Representation of Collections as Maximal Sums

The solution above is similar to taking the meaning of a quantity $\mathrm{K}$ to be that of a maximally-self-connected-portion of $\mathrm{K}$ [17]. However, in the case of collections, topological connection cannot be used as a unifying or characterizing relation to form an integral whole, since collections can easily be spatially scattered. Nonetheless, another type of connection (e.g., social) should always be found. A question begging issue at this point is: why does it seem to be conceptually relevant to find unifying relations leading to (maximal) collections? As discussed in the previous section, collections taken as arbitrary sums of entities make little cognitive sense: we are not interested in the sum of a light bulb, the North Sea, the number 3 and Aida's second act. Instead, we are interested in aggregations of individuals that have a purpose for some cognitive task. So, we require all collectives in our system to form closure systems unified under a proper characterizing relation. For example, a group of visitors of interest can be composed by all those people that are attending a certain museum exhibition at a certain time. Now, by definition, a closure system is maximal (see formula (5)), thus, there can be no group of visitors in this same sense that is part of another group of visitors (i.e., another integral whole unified by the same relation).

Nonetheless, it can be the case that, among the parts of a group of visitors, further structure is obtained by the presence of other collections unified by different relations. For example, it can be the case that among the parts of a group of visitors A, there are collections B and C composed of the English and Dutch speaking people in that group, respectively. Now, neither the English speaking segment nor the Dutch speaking segment are groups of visitors in the technical sense just defined, since the latter has properties lacking in both of them (e.g., the property of having both English and Dutch segments). Moreover, the unifying relations of $\mathrm{B}$ and $\mathrm{C}$ are both specializations of A's unifying relation. For example, $\mathrm{A}$ is the collection of all parties attending an exhibition and the B is the collection of all English speakers among the parties attending that same exhibition. We return to this point in section 4.2.

By not being homeomeros and infinitely divisible, collectives actually bear a stronger similarity to functional complexes than to quantities in the classifications of [10,11]. In [11], for instance, the authors propose that the difference between a collective and a functional complex is that whilst the former has a uniform structure, the 
latter has a heterogeneous and complex one. We propose to rephrase this statement in other terms. In a collective, all member parts play the same role type w.r.t. the whole. For example, all trees in a forest can be said to play the role of a forest member. In complexes, conversely, a variety of roles can be played by different components. For example, if all ships of a fleet are conceptualized as playing solely the role of "member of a fleet" then it can be said to be a collection of ships. Contrariwise, if this role is further specialized in "leading ship", "defense ship", "storage ship" and so forth, the fleet must be conceived as a functional complex.

Finally, we would like to call attention to the fact that collectives are not sets and, thus, the member-collective and the subcollective-collective relations are not the same as the membership $(\in)$ and subset $(\subset)$ relations, respectively. Firstly, collectives and sets belong to different ontological categories: the former are concrete entities that have spatiotemporal features; the latter, in contrast, are abstract entities that are outside space and time and that bear no causal relation to concrete entities [1]. Secondly, unlike sets, collectives do not necessarily obey an extensional principle of identity, i.e., it is not the case that a collective is always completely defined by the sum of its members. We take that some collectives can be considered extensional by certain conceptualizations; however, we also acknowledge the existence of intentional collectives obeying non-extensional principles of identity [21]. Thirdly, collectives are integral wholes unified by proper characterizing relations; sets can be simply postulated by enumerating their members. This feature of the latter is named ontological extravagance and it is a feature to be ruled out from any ontological system [19]. Finally, contrary to sets, we do not admit the existence of empty or unitary collectives. As a consequence, we eliminate a feature of set theory named ontological exuberance [19]. Ontological exuberance refers to the feature of some formal systems that allows for the creation of a multitude of entities without differentiation in content. For instance, in set theory, the elements a, $\{a\},\{\{a\}\},\{\{\{a\}\}\},\{\ldots\{\{\{a\}\}\} \ldots\}$ are all considered to be distinct entities. We shall return to some of these points in the next section.

\section{Parthood Relations Involving Collectives}

\subsection{The Member-Collection Relation}

According to [22], classical semantic analysis of plurals and groups distinguish between atomic entities, which can be singular or collectives, and plural entities. From a linguistic point of view, the member-collection relation is considered to be one that holds between an atomic entity (e.g., John, the deck of cards) and either a plural (e.g., \{John, Marcus\}) or a collective term (e.g., the children of Joseph, the collection of ancient decks).

Before we can continue, a formal qualification of this notion of atomicity is needed. Suppose an integral whole $\mathrm{W}$ unified under a relation R. By using this unifying (or characterizing) relation $\mathrm{R}$, we can then define a composition relation $<_{\mathrm{R}}$ such that $\left(\mathrm{x}<_{\mathrm{R}} \mathrm{W}\right)$ iff: (i) there is a set $\mathrm{B}$ such that $\mathbf{c s}\langle\mathbf{R}\rangle \mathbf{B}$; (iii) $(\mathrm{x}<\mathrm{W})$ and $(\mathrm{x} \in \mathrm{B})$. Intuitively, this relation captures the idea that there is indeed a genuine connection between a part $\mathrm{x}$ and the whole $\mathrm{W}$ (as opposed to a merely formal one). Now, one 
important thing to highlight is that if $\left(\mathrm{x}<{ }_{\mathrm{R}} \mathrm{W}\right)$ then there is no $\mathrm{y}$ such $\left(\mathrm{y}<_{\mathrm{R}} \mathrm{x}\right)$. In other words, the closure set defined by relation $\mathrm{R}$ are the $\mathrm{R}$-atoms of $\mathrm{W}$. This is because, the whole $\mathrm{W}$ unified under $\mathrm{R}$ is maximal under this relation (by the definition of an $\mathrm{R}$ closure system). The fact that no R-part of $\mathrm{W}$ can be unified under the same relation $\mathrm{R}$ of course does not imply that these R-parts need to be atomic in an absolute sense. In fact, given an element $x$ such that $\left(x<_{R} W\right), x$ itself can be an integral wholes unified by a different relation R'. However, it should be clear by now that the sets of R'atoms of $\mathrm{x}$ and the set of $\mathrm{R}$-atoms of $\mathrm{W}$ (of which $\mathrm{x}$ is a member) are disjoint.

An example of a relation that takes place between an atom under relation $\mathrm{R}$ and an integral whole unified under that relation is the member-collective relation (symbolized as $M$ (part,whole)). Following the above discussion, we have that these relations are never transitive, i.e., they are intransitive. Thus, if $\mathrm{M}(\mathrm{x}, \mathrm{W})$ then $\mathrm{x}$ is atomic for $\mathrm{W}$, and if we have $\mathrm{M}(\mathrm{y}, \mathrm{x})$, we also have necessarily that $\neg \mathrm{M}(\mathrm{y}, \mathrm{W})$. In other words, for the case of the member-collective relation, to say that a member must be a singular entity coincides with this entity being an atom in the sense just discussed, i.e., an atom w.r.t. to a characterizing relation unifying that specific whole.

The following example illustrates the intransitivity of the member-collection relation: "I am member of a club $C$ (collective) and my club is a member of an International Association of clubs C' (collective). However, it does not follow that I am a member of this International Association of Clubs $C^{\prime}$ since this only has clubs as members, not individuals". However, an even more general statement about the intransitivity of this relation can be made. Since members of a collective are considered to be atomic w.r.t. the context in which the collective is defined, if an individual $\mathrm{x}$ is a part of (member of) a collection $y$, then for every $z$ which is part of (member of, functional part of, sub-collection of) $\mathrm{x}$, then $\mathrm{z}$ is not a part of (member of) $\mathrm{y}$. In other words, the member-collective relation causes the part to necessarily be seen as atomic in the context of the whole, hence, "blocking" a possible transitive chain of partwhole relations. Thus, for instance, although an individual John can be part of (member of) a Club, none of John's parts (e.g., his heart) is part of that Club.

Regarding the weak supplementation axiom, some authors claim that this axiom is too hard a constraint to be imposed to the member-collective relation [4]. From a formal point of view, this view implies that we accept reflexive characterizing relations for collectives as integral wholes. Such an approach seems at first to be somehow afforded by common sense. For instance, we can conceive a book of poems composed of a single poem, a CD composed of a single track, a purchase order composed of single order item, or a journal issue composed by a single article. Now, are there disadvantages to such an approach? We can foresee two of them.

Firstly, abandoning weak supplementation would set this relation apart from all the other parthood relations that we have considered, since this axiom (considered to be constitutive of the very meaning of part) is assumed by the relations of componentfunctional complex [16], subquantity-quantity [17], and subcollective-collective (section 4.2). Secondly, this choice opens the possibility for the creation of collectives with one single member. But what then would be the difference between John, $\{\mathrm{John}\},\{\{\mathrm{John}\}\},\{\ldots\{\{\mathrm{John}\}\} \ldots\}$, etc? If entities such as these are generally adopted, then our system can face the objection of ontological extravagance, and we should be reminded that avoiding this feature was one of the motivations of mereology in the first place [19]. Given these two reasons, we adopt in this paper the view 
that weak supplementation should be part of the axiomatization of the membercollective relation. This obviously does not imply that we cannot have single-track CD's or single-article journal issues. Following [1], in these cases, we consider the relation between, for instance, the tracks and the CDs to be a relation of constitution as opposed to one of parthood. Relations of constitution abound in ontology. An example is the relation between a marble statue and the (single portion of marble) that constitutes it [1]. In fact, a more detailed analysis of WCH initial proposal showed that some of their original meronymic relations are in fact cases of constitution $[1,11]$.

Finally, let us take the case of the secondary (modal) property of essentiality. As we have previously discussed, unlike sets and mereological sums, collectives do not necessarily have an extensional criterion of identity. That is, whereas for some collectives the addition or subtraction of a member renders a different individual, it is not the case that this holds for all of them. However, when this is the case, all membercollective relations that the extensional collective participates as a whole are relations of essential parthood. This is because, since a collective (by definition) has a uniform structure, then all members of a collective must be indistinguishable w.r.t. the whole. As a consequence, it cannot be the case that some members of a collection are essential while others are not. In summary, member-collective relations are only relations of essential parthood if the collective in the association end connected to the whole is an extensional individual. In the converse reading, if a collective is extensional then all its parts (members) are essential.

\subsection{The Subcollective-Collective Relation}

In contrast with the member-collective relation, from a linguistic point of view, the subcollective-collective is a relation that holds between two plural entities, or collectives constituted by such plural entities, such that all atoms of the first are also atoms of the second [22].

Let us start with an example. Figure 4 depicts an integral whole termed the DSRG (Distributed Systems Research Group) unified by the relation of carrying out research in the area of distributed systems at University $X(U t X)$. The R-atoms of DSRG are them \{John, Mary, Peter, Mark\}. The fact that no R-part of W can be unified under the same relation $\mathrm{R}$ also does not imply that these R-parts cannot be further structured to form new wholes. In other words, for example, we can take two different relations R'and R', which are specializations of R, such that they can be used to form new closure systems among the R-parts of DSRG. Let R' be the relation of carrying out research in the same sub-area of modeling of distributed systems at UtX, and R' be the relation of carrying out research in the same sub-area of performance of distributed systems at UtX. This situation is depicted in figure 5.

Indeed, the fact that $\mathrm{R}^{\prime}$ is a specialization of the condition $\mathrm{R}$ implies that the possible relata of R' are the R-atoms of R, i.e., R' $\subseteq \mathrm{R}$. When this is the case, we name the integral whole $\mathrm{W}^{\prime}$ unified under $\mathrm{R}$ ' a subcollective of the whole $\mathrm{W}$ unified under $\mathrm{R}$. Let us name the relation between $\mathrm{W}^{\prime}$ and $\mathrm{W}$ the relation of subcollective-collective, symbolized as $\mathrm{C}\left(\mathrm{W}^{\prime}, \mathrm{W}\right)$. We then have that $\mathrm{C}\left(\mathrm{W}^{\prime}, \mathrm{W}\right)$ iff: (i) all formal parts of $\mathrm{W}^{\prime}$ are formal parts of $\mathrm{W}$; (ii) the characterizing relation $\mathrm{R}^{\prime}$ of $\mathrm{W}^{\prime}$ is a specialization of

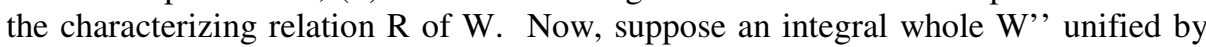
relation $\mathrm{R}^{\prime}$ ' and $\mathrm{C}\left(\mathrm{W}^{\prime}, \mathrm{W}^{\prime}\right)$. By the above definition of the C-parthood relation, we 


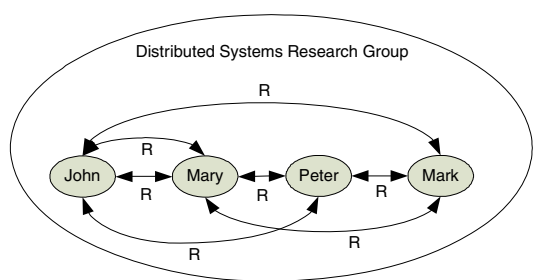

Fig. 4. Examples of an integral whole (collective) and its members

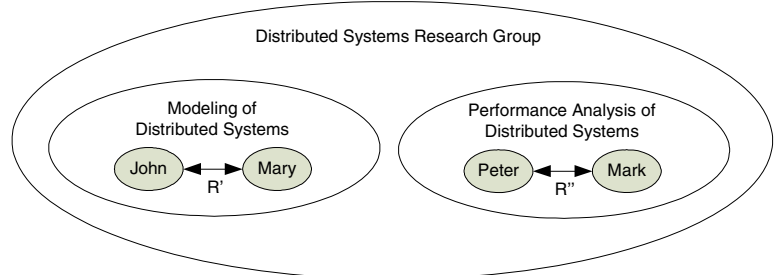

Fig. 5. Examples of a collective and its subcollectives

have that $\mathrm{R}^{\prime} \subseteq \mathrm{R}$ ', and that all parts of $\mathrm{W}$ ', are also parts of $\mathrm{W}$ '. Due to the transitivity of both formal parthood $(<)$ and the subset relation $(\subseteq)$, we have that $\mathrm{R}$ ' $\subseteq \mathrm{R}$, and also that all formal parts of $\mathrm{W}^{\prime}$, are also formal parts of W. Again, by definition, we conclude that $\mathrm{C}\left(\mathrm{W}^{\prime}, \mathrm{W}\right)$ holds. In other words, the subcollective-collective relation is always transitive.

A second property we would like to demonstrate is the following. Suppose we have that $\mathrm{M}(\mathrm{y}, \mathrm{x})$ and $\mathrm{C}(\mathrm{x}, \mathrm{W})$, and that $\mathrm{R}^{\prime}$ and $\mathrm{R}$ are the characterizing relations of $\mathrm{x}$ and $\mathrm{W}$, respectively. Since $\mathrm{M}(\mathrm{y}, \mathrm{x})$, we have both that $(\mathrm{y}<\mathrm{x})$ and that $\mathrm{y}$ is an $\mathrm{R}^{\prime}$-atom of $\mathrm{x}$. From $\mathrm{C}(\mathrm{x}, \mathrm{W})$, we have that all formal parts of $\mathrm{x}$ are formal parts of $\mathrm{W}$, but also that $\mathrm{R}^{\prime} \subseteq \mathrm{R}$. Again, due to the transitivity of both $<$ and $\subseteq$, we have both that $(\mathrm{y}<\mathrm{W})$ and that $\mathrm{y}$ is a R-atom of $\mathrm{W}$. From this, we conclude that $\mathrm{M}(\mathrm{y}, \mathrm{W})$. In other words, transitivity always holds across a member-collective relation combined with a subcollective-collective relation.

Now, how does the subcollective-collective relation stand w.r.t. weak supplementation? Suppose that we have two collectives $\mathrm{x}$ and $\mathrm{y}$ such that $\mathrm{C}(\mathrm{y}, \mathrm{x})$. As we have previously discussed, $\mathrm{x}$ is closure system unified by relation $\mathrm{R}$, and $\mathrm{y}$ must be a closure system unified by a specialization of this condition R'. Since the subcollectivecollective relation is irreflexive, we have that $R^{\prime}$ is necessarily a proper subset of $R$, i.e., there are R-atoms of $\mathrm{x}$ which are not $\mathrm{R}$ '-atoms of $\mathrm{y}$. This, at first, seems to imply that we can always have an integral whole $\mathrm{z}$ which is unified by another specialization R' of R (the complement of R w.r.t. R'). However, the fact that there are members of $\mathrm{X}$ which are not part of $\mathrm{y}$ does not mean that these members can define a genuine integral whole. In other words, it can be the case that the only relation in common between these entities is that they obey the condition implied by $\mathrm{R}$ and the negation the condition implied by R'. As discussed in [1,5], characterizing entities based on negative properties is a poor ontological choice. For this reason, if we have that $\mathrm{C}(\mathrm{y}, \mathrm{x})$ we do not require that there is a $\mathrm{z}$ different from $\mathrm{y}$ such that $\mathrm{C}(\mathrm{z}, \mathrm{x})$, but we do require that there is a $\mathrm{z}$ such that $\neg \operatorname{overlap}(\mathrm{z}, \mathrm{y})$ and $\mathrm{M}(\mathrm{z}, \mathrm{x})$. 
In the previous sections, we have discussed that collectives are not necessarily extensional and that, if a member of a collective is essential to the collective, then the collective is an extensional entity, i.e., all its members are essential. How do subcollectives stand w.r.t. to this secondary property? Suppose that we have a collective W composed of the subcollective W' and W', such that the former is an essential part of W but not the latter. Now, as we have discussed, the structure of collectives is defined via specialization of the collective's unifying relation, i.e., the members of $\mathrm{W}^{\prime}$ and $\mathrm{W}$ ' are also members of $\mathrm{W}$. This implies that all subcollectives of $\mathrm{W}$ are inseparable parts of it, i.e., W' and $\mathrm{W}^{\prime}$ ' come to existence by refining the structure of $\mathrm{W}$ and by grouping the specific members of $\mathrm{W}$. As a consequence, they cannot exist without that whole. A second observation we can make is that if there is an $\mathrm{x}$ which is a member of $\mathrm{W}^{\prime}$, and $\mathrm{x}$ is an essential member of $\mathrm{W}$ then $\mathrm{x}$ must also be an essential member of $\mathrm{W}^{\prime}$. The argument can be made as follows. If $\mathrm{x}$ is an essential part of $\mathrm{W}$ then $\mathrm{W}$ cannot exist without $\mathrm{x}$; If $\mathrm{W}^{\prime}$ ' is an inseparable part of $\mathrm{W}$ then $\mathrm{W}^{\prime}$ cannot exist without $\mathrm{W}$; due to the transitivity of existential dependence [14], we have that $\mathrm{W}^{\prime}$ cannot exist without $\mathrm{x}$, ergo, $\mathrm{x}$ is an essential part of $\mathrm{W}^{\prime}$. Finally, since we are admitting that collectives are not necessarily extensional entities, it is conceivable that a whole $\mathrm{W}$ has an essential part $W^{\prime}$ composed of members which are not essential for either $\mathrm{W}$ or W'. For instance, suppose that by law, all juries must have at least two members which are older than sixty years old. Although this subcollective would be essential to the whole, it is conceivable that its individual members are exchangeable. By the same reasoning, one could admit a particular subcollective to be essential to a whole, without requiring the other collectives of that whole to be likewise essential.

\subsection{Towards a UML Profile for Modeling Collectives and Their Parts}

We summarize the results of these sections in a proposal that has been incorporated in a UML profile for representing the member-collective and the subcollective-collective relations (table 1). Since a profile is constituted by syntactical constraints and, since UML conceptual models are always defined at the type level, the meta-properties of irreflexivity, anti-symmetry and transitivity (at instance level) cannot be captured by profile constraints. We have included a constraint to guarantee weak supplementation for these relations taking into consideration the type-level nature of a UML class diagram, i.e., taking into consideration the minimum cardinality constraints of all parthood relations connected to the same type representing a whole.

\begin{tabular}{|cl|}
\hline $\begin{array}{c}\text { Metaclass } \\
\text { A }\end{array}$ & $\begin{array}{l}\text { Description } \\
\text { A «collective» represents a type whose instances are collectives, i.e., they are } \\
\text { collections of entities that have a uniform structure. Examples include a deck } \\
\text { of cards, a forest, a group of visitors, a pile of bricks. }\end{array}$ \\
\hline $\begin{array}{c}\text { subcollective- } \\
\text { collective }\end{array}$ & $\begin{array}{l}\text { This parthood relation holds between two collectives. Examples include: (a) } \\
\text { the north part of the Black Forest is part of the Black Forest; (b) The collec- } \\
\text { tion of Jokers in a deck of cards is part of that deck; (c) the collection of } \\
\text { forks in a cutlery set is part of that cutlery set. We use the symbols }\end{array}$ \\
& $\begin{array}{l}\text { s } \\
\text { shareable (see http://www.uml.org/) versions of this relation, respectively. }\end{array}$ \\
\end{tabular}




\begin{tabular}{|ll|}
\hline $\begin{array}{l}\text { member- } \\
\text { collective }\end{array}$ & $\begin{array}{l}\text { This is a parthood relation between a functional complex or a collective (as a } \\
\text { part) and a collective (as a whole). Examples include: (a) a tree is part of } \\
\text { forest; (b) a card is part of a deck of cards; (c) a club member is part of a } \\
\text { club. We use the symbols } \\
\text { shareable and non-shareable versions of this relation, respectively. }\end{array}$ \\
\hline General Constraints
\end{tabular}

1. Weak Supplementation: Let $T$ be a type whose instances are wholes and let $\left\{T_{1} \ldots T_{2}\right\}$ be a set of types related to $\mathrm{T}$ via the subcollective-collective or member-collective relations. Let lower $\mathrm{Ci}_{\mathrm{C}}$ be the value of the minimum cardinality constraint of the association end connected to $\mathrm{C}_{\mathrm{i}}$ in the aggregation relation. Then, we have that

$$
\left(\sum_{i=1}^{n} \operatorname{lower}_{\mathrm{Ci}}\right) \geq 2 \text {; }
$$

\section{Constraints applied to the subcollective-collective relation}

1. This relation only holds between collectives, i.e., they must be either stereotyped as «collective» or be a subtype of a type stereotyped as «collective»;

2. Collectives are maximal entities. For this reason, it is not the case that a collective can have as a part another collective of the same type (i.e., unified by the same relation). As a consequence, these relations are irreflexive at the type level. In UML terms, the two association ends of this relation must be connected to classes of different types (albeit both stereotyped as «collective»);

3. Also because collectives are maximal entities, a collective can have at maximum one subcollective of a given type. For this reason, the maximum cardinality constraint in the association end connected to the part in this relation must be one (in UML terms, self.target.upper $=1$ );

4. All subcollective-collective relations are relations of inseparable parthood. These relations are marked with a tagged value \{insperable\} and the association end connected to the whole must be immutable (in UML terms, self.source.readOnly = true);

5. This relation conforms to the axiomatization of Minimum Mereology (MM), i.e., it is an Irreflexive, Asymmetric and Transitivity relation which obeys the Weak Supplementation axiom. Moreover, if a collective $\mathrm{W}$ has one single direct subcollective $\mathrm{W}^{\prime}$, then it must have members which are disjoint from W';

\section{Constraints applied to the member-collective relation}

1. This relation can only represent essential parthood if the object representing the whole on this relation is an extensional individual. In this case, all parthood relations in which this individual participates as a whole are essential parthood relations. These relations are marked with tagged value \{essential\} and the association end connected to the part must be immutable (in UML terms, self.target.readOnly = true);

2. The class connected to association end relative to the whole individual must be a type whose instances are collectives, i.e., they must be either stereotyped as «collective» or be a subtype of a type stereotyped as «collective»;

3. This is an Irreflexive and Asymmetric relation which obeys the Weak Supplementation axiom. However, it is also an Intransitive relation. Although transitivity does not hold across two member-collective relations, a member-collective relation followed by 
subcollective-collective relation is transitive. That is, for all a,b,c, if $\mathrm{M}(\mathrm{a}, \mathrm{b})$ and $\mathrm{M}(\mathrm{b}, \mathrm{c})$ then $\neg \mathrm{M}(\mathrm{a}, \mathrm{c})$, but if $\mathrm{M}(\mathrm{a}, \mathrm{b})$ and $\mathrm{C}(\mathrm{b}, \mathrm{c})$ then $\mathrm{M}(\mathrm{a}, \mathrm{c})$.

4. Asides from being intransitive, a member $\mathrm{x}$ of a collective $\mathrm{W}$ is atomic w.r.t. the collective. This means that for if an entity $y$ is part of $x$ then $y$ is not a member of $W$.

For the sake of illustration, we revisit in figures 6.a and 6.b two of the examples discussed in this paper, explicitly representing them with the modeling primitives proposed in table 1 . As one can observe, we decorate the standard UML symbol for aggregation with a $\mathrm{C}$ and an $\mathrm{M}$ to represent a subcollective-collective and membercollective relations, respectively.

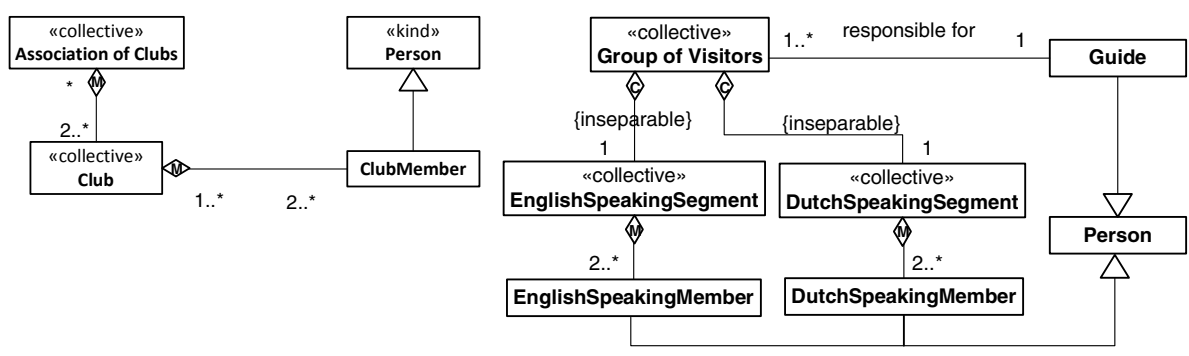

Fig. 6. Examples of subcollective-collective and member-collective part-whole relations

\section{Final Considerations}

The development of suitable foundational theories is an important step towards the definition of precise real-world semantics and sound methodological principles for conceptual modeling languages. This article concludes a sequence of papers that aim at addressing the three fundamental types of wholes prescribed by theories in linguistics and cognitive sciences, namely, functional complexes, quantities, and collectives. The first of these roughly correspond to our common sense notion of object and, hence, the standard interpretation of objects (or entities) in the conceptual modeling literature is one of functional complexes. The latter two categories, in contrast, have traditionally been neglected both in conceptual modeling as well as in the ontological analyzes of conceptual modeling grammars.

In this paper, we conduct one such ontological analysis to investigate the proper representation of types whose instances are collectives, as well as the representation of parthood relations involving them. As result, we were able to provide a sound ontological interpretation for these notions, as well as modeling guidelines for their proper representation in conceptual modeling. In addition, we have managed to provide a precise qualification for the relations of member-collective and subcollectivecollective w.r.t. to both classical mereological properties (e.g., transitivity, weak supplementation, extensionality) as well as modal secondary properties that differentiate essential and inseparable parts. Finally, the results advanced here contribute to the definition of concrete engineering tools for the practice of conceptual modeling. In 
particular, the metamodel extensions and associated constraints outlined here have been implemented in a Model-Driven Editor using available UML metamodeling tools [23].

Acknowledgements. This is research is funded by FAPES (grant \# 45444080/09) and CNPq (grant \# 481906/2009-6) as well as a CNPq research productivity grant.

\section{References}

[1] Guizzardi, G.: Ontological Foundations for Structural Conceptual Models, Telematica Institute Fundamental Research Series, The Netherlands (2005) ISBN 90-75176-81-3

[2] Guizzardi, G.: Modal aspects of object types and part-whole relations and the shape de re/de dicto distinction. In: Krogstie, J., Opdahl, A.L., Sindre, G. (eds.) CAiSE 2007 and WES 2007. LNCS, vol. 4495, pp. 5-20. Springer, Heidelberg (2007)

[3] Rector, A., Rogers, J., Bittner, T.: Granularity, scale and collectivity: When size does and does not matter. Journal of Biomedical Informatics 39(3), 333-349 (2006)

[4] Bittner, R., Donelly, M., Smith, B.: Individuals, Universals, Collections: On the Foundational Relations of Ontology. In: 3rd Intl. Conf. on Formal Ontology in Inf. Systems (FOIS 2004), Torino, Italy (2004)

[5] Bunge, M., Ontology I.: The Furniture of the World. Springer, Heidelberg (1977)

[6] Opdahl, A., Henderson-Sellers, B., Barbier, F.: Ontological Analysis of whole-part relationships in OO-models. Information and Software Technology 43, 387-399 (2001)

[7] Wand, Y., Storey, V.C., Weber, R.: An ontological analysis of the relationship construct in conceptual modeling. ACM Transactions on Database Systems 24(4), 494-528 (1999)

[8] Winston, M.E., Chaffin, R., Herrman, D.: A Taxonomy of Part-Whole relations. Cognitive Science (1987)

[9] Odell, J.J.: Six Different Kinds of Composition. Journal of Object-Oriented Programming 5/8 (1994)

[10] Pribbenow, S.: Meronymic Relationships: From Classical Mereology to Complex PartWhole Relations, The Semantics of Relationships. Kluwer Academic Publishers, Dordrecht (2002)

[11] Gerstl, P., Pribbenow, S.: Midwinters, End Games, and Bodyparts. A Classification of Part-Whole Relations. Intl. Journal of Human-Computer Studies 43, 865-889 (1995)

[12] Bittner, T., Donnelly, M.: Logical properties of foundational relations in bio-ontologies. Artificial Intelligence in Medicine 39, 197-216 (2007)

[13] Keet, C.M., Artale, A.: Representing and Reasoning over a Taxonomy of Part-Whole Relations. Applied Ontology 3(1-2), 91-110 (2008)

[14] Simons, P.M.: Parts. An Essay in Ontology. Clarendon Press, Oxford (1987)

[15] Gangemi, A., Guarino, N., Masolo, C., Oltramari, A.: Understanding top-level ontological distinction. In: Proceedings of IJCAI 2001, Workshop on Ontologies and Information Sharing (2001)

[16] Guizzardi, G.: The problem of transitivity of part-whole relations in conceptual modeling revisited. In: van Eck, P., Gordijn, J., Wieringa, R. (eds.) CAiSE 2009. LNCS, vol. 5565, pp. 94-109. Springer, Heidelberg (2009)

[17] Guizzardi, G.: On the Representation of Quantities and their Parts in Conceptual Modeling. In: 6th International Conf. on Formal Ontology and Information Systems (FOIS 2010), Toronto, Canada (2010) 
[18] Guizzardi, G.: Representation of Collectives and their Members in UML Conceptual Models: An Ontological Analysis. In: Proc. of the 6th FP-UML International Workshop, Vancouver, Canada (2010)

[19] Varzi, A.C.: Parts, wholes, and part-whole relations: The prospects of mereotopology. Journal of Data and Knowledge Engineering 20, 259-286 (1996)

[20] Cadoli, M., Calvanese, D., De Giacomo, G., Mancini, T.: Finite satisfiability of UML class diagrams by constraint programming. In: Workshop on CSP Techniques with Immediate Application (2004)

[21] Botazzi, E., Catenacci, C., Gangemi, A., Lehmann, J.: From Collective Intentionality to Intentional Collectives: An Ontological Perspective, Cognitive Systems Research, Special Issue on Cognition and Collective Intentionality (2006)

[22] Vieu, L., Aurnague, M.: Part-of Relations, Functionality and Dependence, Categorization of Spatial Entities in Language and Cognition. John Benjamins, Amsterdam (2007)

[23] Benevides, A.B., Guizzardi, G.: A model-based tool for conceptual modeling and domain ontology engineering in ontoUML. In: Filipe, J., Cordeiro, J. (eds.) Enterprise Information Systems. Lecture Notes in Business Information Processing, vol. 24, pp. 528-538. Springer, Heidelberg (2009) 